\title{
Natural occurrence of the diamond hexagonal structure in silicon nanowires grown by a plasma assisted vapour-liquid-solid method
}

\author{
J. Tang ${ }^{a}$, J.-L. Maurice ${ }^{a}{ }^{\dagger}$, F. Fossard ${ }^{b}$, I. Florea ${ }^{a}$, W. Chen ${ }^{a}$, E. V. Johnson ${ }^{a}$, M. Foldyna ${ }^{a}$, L. Yu ${ }^{c}$ and P. \\ Roca i Cabarrocas ${ }^{a}$
}

Silicon nanowires have been grown by a plasma-assisted vapourliquid-solid method using tin as the catalyst. Transmission electron microscopy in the [1-210] zone axis shows that the diamond hexagonal $\left(\mathrm{PG}_{3} / \mathrm{mmc}\right)$ crystal structure is present in several nanowires. This is the first unambiguous proof of the natural occurrence of this metastable phase to our knowledge.

\section{Introduction}

In standard (pressure, temperature) conditions, silicon has a diamond cubic (dc, Fd-3m) crystalline structure. Its properties such as the bandgap, carrier mobility and absorption are specific to that structure. The diamond cubic arrangement is a face-centred cubic ( $\mathrm{fcc}$ ) structure with a two-atom motif. It is one close-packed stacking arrangement, called polytype $3 \mathrm{C}$, of which there are many other polytypes. Most of these polytypes have hexagonal symmetry $\mathrm{P}_{3} / \mathrm{mmc}$; they correspond to hexagonal close packed (hcp) phases. In silicon, these polytypes are thus possible and would present other electronic band structures with different band gaps. The calculated band structure of the diamond hexagonal $2 \mathrm{H}$ polytype of $\mathrm{Si}$ indicates that it would have a smaller band gap that would become direct in nanowires ${ }^{1}$ or under strain ${ }^{2}$. Interestingly, it would provide a higher absorption of the solar spectrum $^{1,2}$. In silicon, these polytypes are metastable and are not present in the phase diagram ${ }^{3-5}$. Names for the diamond hexagonal phase include $2 \mathrm{H}$, dh, hd and lonsdaleite; the term "wurtzite", which is often found in the literature, is actually for cases where the two atoms of the motif are different (e.g. $\mathrm{GaN}$ ). The first report of the $2 \mathrm{H}$ polytype was in 1963 by Wentorf et $\mathrm{al}^{6}$. These authors obtained the $2 \mathrm{H}$ phase by applying a series of high pressure ( 15 to $16 \mathrm{GPa})$ and high temperature $\left(200^{\circ} \mathrm{C}\right.$ to $\left.1000^{\circ} \mathrm{C}\right)$ treatments to diamond cubic

\footnotetext{
a. LPICM, CNRS, Ecole polytechnique, Université Paris-Saclay, 91128 Palaiseau, France.

b. LEM, CNRS, ONERA, 92322 Châtillon, France.

c. School of Electronics Science and Engineering, Nanjing University, Nanjing 210093, China.

† jean-luc.maurice@polytechnique.edu
}

crystalline $\mathrm{Si}$. In the literature, there are many theoretical predictions of such hexagonal Si phase ${ }^{7-11}$, but few examples of experimental evidence ${ }^{12-14}$, in addition to some examples for $\mathrm{Ge}$ or $\mathrm{SiGe} \mathrm{e}^{15,16}$. It is quite remarkable that apart from the cases where it is obtained by the use of an epitaxial template ${ }^{14,16}$, the $2 \mathrm{H}$ polytype occurs in cases where a shear stress has been applied $^{12,13,15}$. Other reports show that $2 \mathrm{H}$ SiNWs can be grown directly ${ }^{17-20}$. However, the proofs in these reports are given by transmission electron microscopy (TEM) analyses carried out in such a way that cubic Si multiple twinning would exhibit a very similar contrast ${ }^{21,22}$. Therefore, there remains a lingering uncertainty about whether these objects are truly hexagonal, and therefore about the very possibility of directly growing this metastable phase in nanowires.

The difference between the types of stacking of close packed planes (which defines polytypism) is only directly visible in one type of zone axis: $[110]_{3 \mathrm{C}} /[1-210]_{2 \mathrm{H}}$. Any other zone axis will give ambiguous results. In this paper, we present TEM characterizations in the $[1-210]_{2 \mathrm{H}}$ zone axis, in cases where cubic multiple twinning cannot be mistaken for diamond hexagonal stacking. This work is thus the first unambiguous demonstration of the natural ${ }^{\ddagger}$ occurrence of the metastable diamond hexagonal structure. The $2 \mathrm{H}$ phase appears to be quite stable: it remained after 5 months in air, and after annealing at $700^{\circ} \mathrm{C}$.

\section{Experimental details}

Silicon nanowires were grown through a plasma-assisted vapour-liquid-solid (VLS) method using a Sn catalyst ${ }^{23,24}$. In order to observe the crystallinity of the SiNWs without any transfer process, they were grown directly on carbon-coated TEM grids. First, a $\mathrm{Cu}$ grid was attached to a piece of $\mathrm{ZnO}$ :Al coated Corning glass using silver paste (Fig. 1a). Then a 1-nm thick Sn film was thermally evaporated on top. The substrate was then loaded into a standard plasma-enhanced chemical vapour deposition (PECVD) reactor to perform the SiNW growth. During the growth, the gas pressure, substrate temperature, radio frequency (RF) plasma power density and 

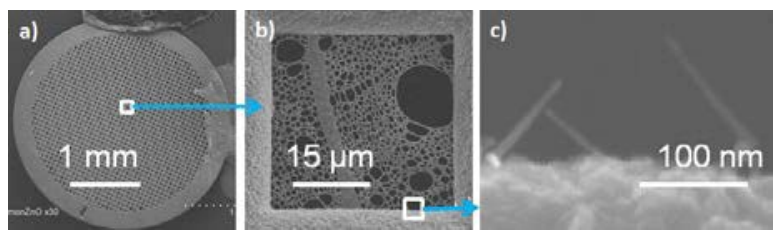

FIG. 1. a) SEM image of TEM grid; b) detail of (a); c) SiNWs grown on sidewall of CU TEM grid.

the growth duration were $133 \mathrm{~Pa}, 400^{\circ} \mathrm{C}, 17 \mathrm{~mW} / \mathrm{cm}^{2}$ and 2 min, respectively. After the growth, the sample was first analysed by scanning electron microscopy (SEM) (Fig. 1). Then the $\mathrm{CU}$ TEM grid was removed from the substrate and loaded into a TEM microscope for high-resolution imaging. The SEM equipment was a Hitachi S-4800, and the TEM instruments were a Jeol 2010F with a point-to-point resolution of $0.23 \mathrm{~nm}$, and a Zeiss Libra $200 \mathrm{FE}$, with a point-to-point resolution of $0.24 \mathrm{~nm}$. In situ annealing was performed in the latter, using a Gatan heating holder, with plateaus at $230^{\circ} \mathrm{C}(51 \mathrm{~min}) 240^{\circ} \mathrm{C}$ $(25 \mathrm{~min}), 470^{\circ} \mathrm{C}(25 \mathrm{~min}), 600^{\circ} \mathrm{C}(9 \mathrm{~min})$ and $700^{\circ} \mathrm{C}(5 \mathrm{~min})$. The acceleration voltage was $15 \mathrm{kV}$ for the SEM, and $200 \mathrm{kV}$ for both the TEMs.

\section{Results}

All the observed hexagonal SiNWs were grown directly on the $\mathrm{CU}$ TEM grid. During the TEM observations, we found seven nanowires oriented close to a $[110]_{3 \mathrm{C}} /[1-210]_{2 \mathrm{H}}$ zone axis, the only orientation where $2 \mathrm{H}$ characterisation can be done. Among those seven, five contained significant sections having the $2 \mathrm{H}$ structure. These five NWs are the subjects of the following analysis.

A high resolution TEM image (HRTEM) of a SiNW grown on the Cu TEM grid is shown in Fig. 2a. It has been recorded in the $[110]_{3 \mathrm{C}} /[1-210]_{2 \mathrm{H}}$ zone axis, so that the stacking sequence of the $(111)_{3 \mathrm{C}} /(0002)_{2 \mathrm{H}}$ close-packed planes is directly visible. In

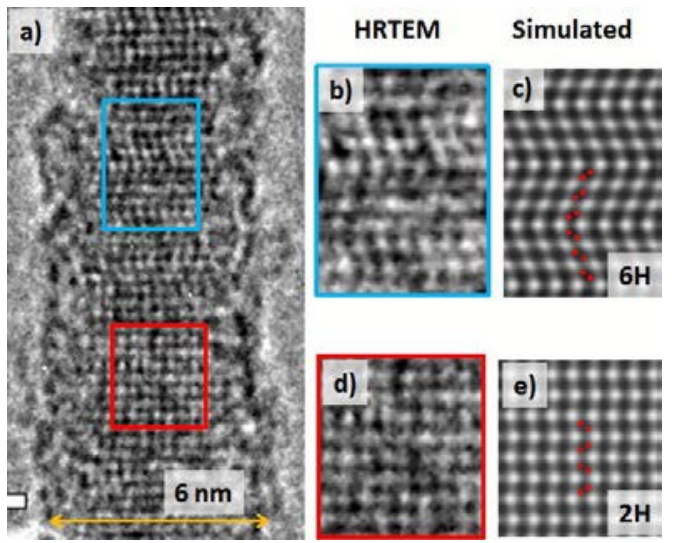

FIG. 2. TEM characterization of hexagonal SiNW. a) HRTEM image of the SiNWs in a [1-210 $]_{2 \mathrm{H}}$ zone axis; b) and d) are zooms of the indicated areas; c) and e) are simulated $6 \mathrm{H}$ and $2 \mathrm{H}$ Si HRTEM images, respectively; the red dots in c) and $\mathrm{e}$ ) indicate the positions of the atomic columns. order to strengthen this direct evidence, HRTEM simulations were carried out using the JEMS package ${ }^{25}$. The thickness in these simulations was taken approximately equal to the crystalline SiNW core diameter: $4.6 \mathrm{~nm}$; the best fit between the experimental and simulated images was obtained with a defocus of $60 \mathrm{~nm}$ (Scherzer defocus was $54 \mathrm{~nm}$ ); in this case, each dark dot corresponds to two columns of Si atoms, as shown in Figs. $2 c$ and 2 e. The enlarged Fig. $2 b$ shows the top part of this NW. It can be seen that the dark dots have a translational symmetry with a periodicity of six along the vertical direction. This structure is consistent with the $6 \mathrm{H}$ polytype, and shows good agreement with the simulated $6 \mathrm{H} \mathrm{Si}$ structure shown in Fig. 2c. The enlarged Fig. $2 \mathrm{~d}$ shows the bottom part of this NW; it can be seen that the dark dots are now almost aligned in the vertical direction. In fact these dots have a translational symmetry with a periodicity of 2 along the vertical direction, consistent with a $2 \mathrm{H}$ polytype $^{3}$, and it also shows good agreement with the simulated $2 \mathrm{H}$ structure shown in Fig. 2e. The amorphous contrast at the NW surface indicates the presence of an oxide shell.

The pure $2 \mathrm{H}$ structure has also been observed in other nanowires grown in the same batch. Figure 3 shows a NW with $90^{\circ}$ change in the growth direction. The HRTEM image of the bottom part of this NW is shown in Fig. 3a, with the corresponding FFT shown in the inset. Both the HRTEM image and the FFT pattern show that the NW has a $2 \mathrm{H}$ arrangement.
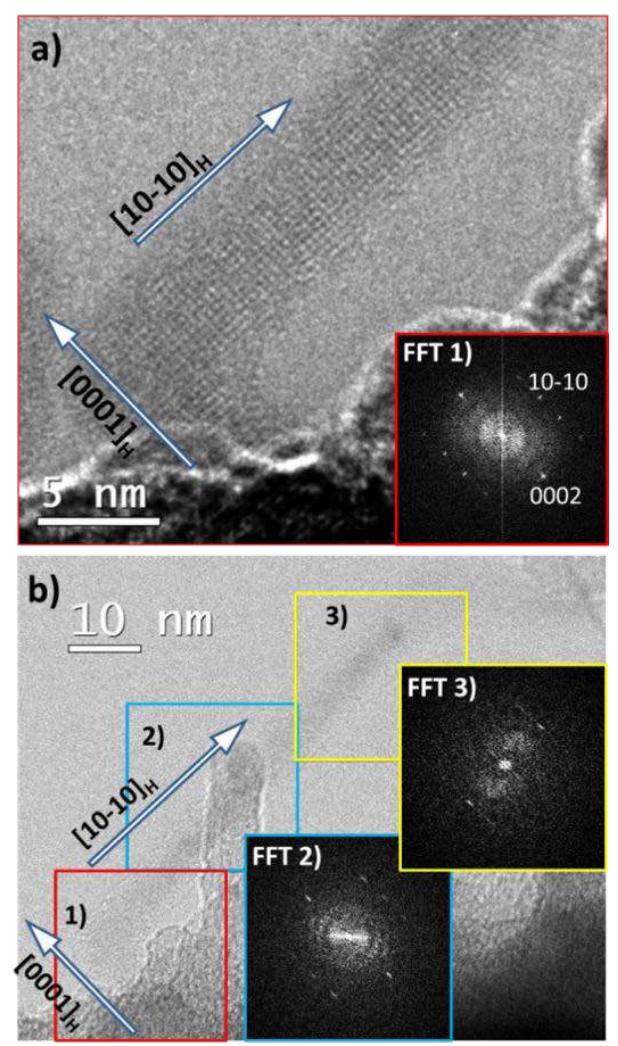

FIG. 3. HRTEM images of pure phase $2 \mathrm{H}$ SiNW. a) The bottom part of this NW, with a FFT of this image shown in the inset. b) Full view of the NW, the two insets are the FFTs of the indicated regions. 


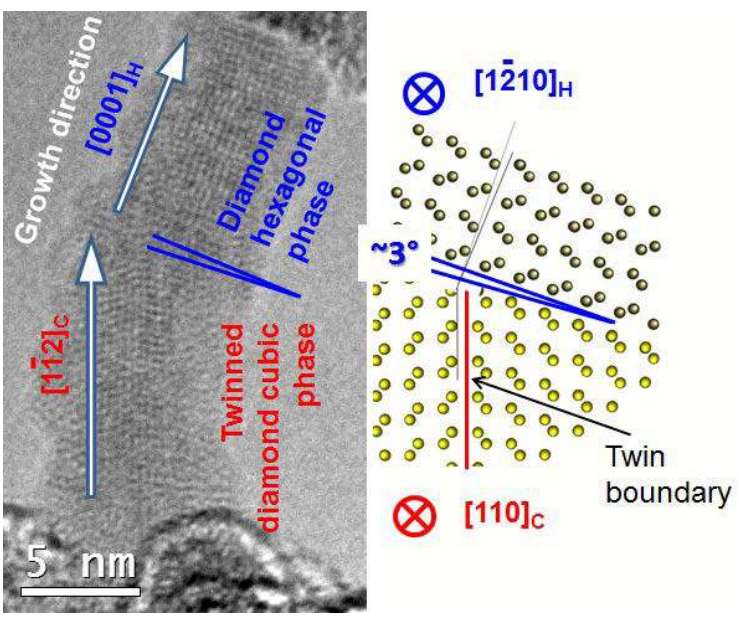

FIG. 4. Switch from twinned cubic phase to $2 \mathrm{H}$ phase, associated with a change in growth direction. The schematic shows that the relationship between the two phases is close to epitaxial.

The NW growth direction has changed from [0001] to [10-10] with no visible crystalline defects. In Fig. $3 b$, the FFT of the middle and top part of the NW are shown in the insets. Even if these FFT patterns are less clear than that of the bottom part of the NW, they all have the same geometry. This indicates that the whole NW has the $2 \mathrm{H}$ structure.

In figure 4, the NW has a diamond cubic phase near its base and a $2 \mathrm{H}$ phase at its top. In the cubic part, there is a twin plane, as shown in the schematic on the right of Fig. 4 . The first layer of the $2 \mathrm{H}$ part may have a twin relation with the cubic part. There is a $\sim 3^{\circ}$ misorientation between the actual orientation of the $2 \mathrm{H}$ part and what would be expected from a perfect twin orientation. This switching from the diamond cubic phase to the $2 \mathrm{H}$ polytype would indicate that the latter was energetically favourable at this stage of growth, under the precise growth conditions that were present.

In order to study the stability of the hexagonal SiNWs, we have kept the SiNW sample under standard ambient temperature and pressure conditions for 5 months before further TEM observation. Figure 5 a shows the TEM image of such a SiNW. This NW has a diameter around $8 \mathrm{~nm}$ at its base and a length of $\sim 90 \mathrm{~nm}$. The enlarged Fig. $5 \mathrm{~b}$ shows the crystallinity of the NW. It can be seen that a thick $(\sim 3 \mathrm{~nm})$ amorphous SiOx has developed at the surface of this NW, but the core part has kept a $2 \mathrm{H}$ crystalline structure. Aligning the zone axis was delicate as the nanowire moved under electron beam excitation. Thus, the electron diffraction pattern (EDP) recorded in selected area mode $(65 \mathrm{~nm})$ lacks several spots (fig. 5c); however, it clearly exhibits $2 \mathrm{H}$ diffraction spots that cannot be interpreted as originating from cubic stacking. This shows that the $2 \mathrm{H}$ phase in such thin nanowires is stable under standard atmosphere conditions.

The thermal stability of $2 \mathrm{H}$ SiNW was also studied. After acquiring the HRTEM image of the NW in Fig. 5a, the temperature of the TEM grid holder has been increased to anneal the NW and determine the temperature up to which

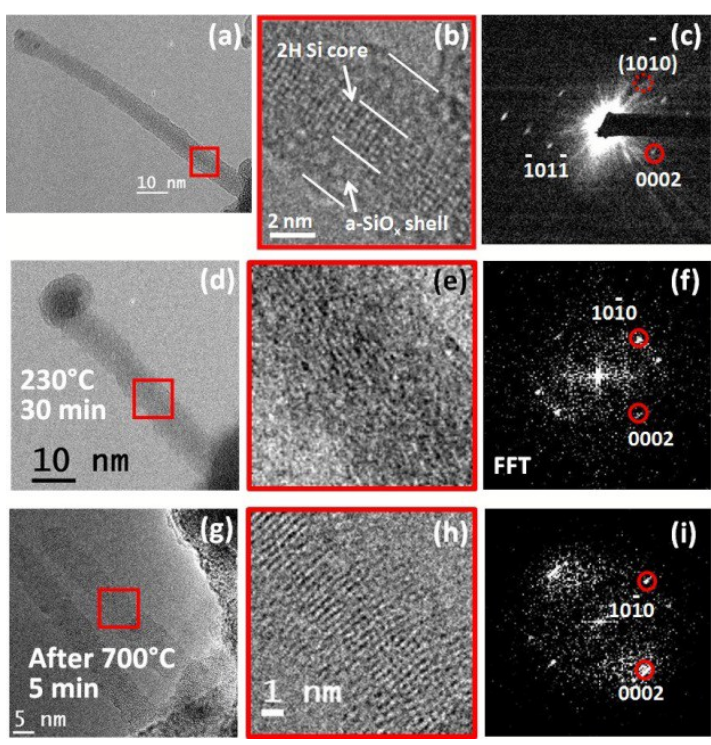

FIG. 5. (a-f) $2 \mathrm{H}$ SiNW that has been kept in standard atmosphere condition for 5 months. a) TEM image, b) enlargement of red square in (a), c) selected area (65 nm) EDP. d) Same SiNW after 30 minutes of annealing at $230^{\circ} \mathrm{C}$. e) Enlargement of red square in (d); f) FFT of the image in e). (g-i) Similar nanowire but not irradiated during heating, having been additionally annealed in situ up to $700^{\circ} \mathrm{C}$ for 5 min (image recorded at room temperature after cooling). h) Enlargement of red square in (g); i) FFT of the image in (h).

the $2 \mathrm{H}$ phase is stable. Figure $5 \mathrm{~d}$ shows the NW after 30 minutes of annealing at $230^{\circ} \mathrm{C}$. This is the same NW as the one shown in Fig. 5a. The NW has started to bend; however, a FFT of the enlargement shown in Fig. 5e still reveals the presence of $2 \mathrm{H}$ spots, indicating that the phase is stable up to that temperature. Unfortunately, the [1-210] zone axis is lost after further bending induced by electron irradiation, which stops the observation of the phase at higher temperatures. However, we were able to record, at room temperature, the image of another nanowire, which had not been irradiated during in situ annealing (fig. $5 \mathrm{~g}-\mathrm{i}$ ). That nanowire had thus undergone the whole annealing cycle up to $700^{\circ} \mathrm{C}$ (see experimental section). The FFT of its core (fig. 5i) still exhibits $2 \mathrm{H}$ spots. This nanowire must have kept its $2 \mathrm{H}$ polytype up to $700^{\circ} \mathrm{C}$ or, alternatively, it must have recovered it upon cooling. Either explanation demonstrates that the $2 \mathrm{H}$ phase is relatively robust in such objects and benefits from a significant stability domain.

In the following, we extract as much quantitative data as possible from the TEM measurements. Table I summarises the geometrical properties of the $2 \mathrm{H}$ nanowires and shows their $d_{10-10}$ interplanar spacings and the angles between their (0002) and (10-10) planes, as measured in FFTs and diffraction patterns. A total of twenty-two measurements were performed on the five nanowires.

These measurements depend on the equipment used, and variations of $-4 \%$ to $+6 \%$ were observed with respect to the mean. Even with the same microscope, variations between different magnifications in imaging mode and between 
imaging and diffraction modes reached $3 \%$ on the same object, due to different calibrations and varying departures from the exact [1-210] zone axis. The averaged $d_{0002}$ was $0.3143 \mathrm{~nm}$, quite close to the bulk value $d_{0002}=0.3162 \mathrm{~nm}$ calculated by Rödl et al. for unstressed $2 \mathrm{H}-\mathrm{Si}^{2}$. Thus we decided to normalise all measurements to this reference value, so that only $d_{10-10}$ varies in table I. We estimate the relative measurement error for $d_{10-10}$ to be within +/- $0.3 \%$. Quite remarkably, measurements performed in the two differently oriented regions ([0002] and [10-10]) of the NW in fig. 3 exhibited no difference in parameters, suggesting that the surface geometry (and the pressure applied by surface tension) played no role. We also measured the angle between these two families of planes. This angle varied slightly from one measurement to the next on the same object, probably due to the rapidly varying orientation with respect to the beam, and because of the elongation of the spots perpendicular to the wire axis. Therefore, we estimate the uncertainty to be about $+/-1.5^{\circ}$. Except for a value of $85^{\circ}$ in the case of the NW of fig. $5 a-f$, all measurements were equal within that margin of error to $90^{\circ}$. We attribute the value of $85^{\circ}$ to the bending of that particular NW, as its crystalline core was only $2 \mathrm{~nm}$ in diameter (see fig. 5a-b). All the angles were arbitrarily taken $<90^{\circ}$ because one cannot differentiate 10-10 from -1010.

The theoretical bulk values for $2 \mathrm{H} \mathrm{Si}$ in Table 1 were derived using the crystal parameters calculated by Rödl et $a l^{2}{ }^{2}$ : $a=0.3828 \mathrm{~nm}, c=06325 \mathrm{~nm}$. The experimental values in Table I are quite close to these values: the average measured $d_{10}$ ${ }_{10} / d_{0002}$ ratio is $1.3 \%$ greater than the theoretical value for $2 \mathrm{H}$ $\mathrm{Si}$, and (neglecting the $85^{\circ}$ of the NW of fig. $5 \mathrm{a}-\mathrm{f}$ ) the average angle is only $1.2 \%$ off. These results show that indeed, the nanowires presented here possess the $2 \mathrm{H}$ hexagonal structure, either partly or completely.

Finally, given the importance of the exact composition of the catalyst and of the impurity content in the silicon, we have performed energy-filtered TEM and energy-dispersive X-ray microanalysis (EDX) on some of the NWs. However, the chemical analysis of such small objects is difficult. To get a significant signal from $\mathrm{Cu}$, the energy-filtered TEM method required an energetic electron dose that destroyed the NWs, making the analysis impossible. EDX, on the other hand, delivered an intense $\mathrm{Cu}$ signal everywhere on the objects

Table I: Properties as measured by TEM for the NWs shown in figs. 2 to 5 , compared to theoretical values ${ }^{2}$. See measurement errors in text.

\begin{tabular}{|c|c|c|c|c|c|c|}
\hline $\begin{array}{c}\text { Nano } \\
\text { wire }\end{array}$ & $\begin{array}{c}\text { Length } \\
(\mathrm{nm})\end{array}$ & $\begin{array}{c}\text { Crystalline } \\
\text { diameter } \\
(\mathrm{nm})\end{array}$ & $\begin{array}{c}\text { Per- } \\
\text { cent } 2 \mathrm{H}\end{array}$ & $\begin{array}{l}d_{10-10} \\
(\mathrm{~nm})\end{array}$ & $\begin{array}{l}d_{10-10} \\
/ d_{0002} \\
\text { Ratio }\end{array}$ & $\begin{array}{c}(10-10)- \\
(0002) \\
\text { Angle }\left(^{\circ}\right)\end{array}$ \\
\hline Fig2 & 6 & 3.3 & 40 & 0.337 & 1.069 & 88.8 \\
\hline Fig3 & 95 & 4 & 100 & 0.339 & 1.076 & 89.1 \\
\hline Fig4 & 100 & 4 & 70 & 0.340 & 1.078 & 89.5 \\
\hline Fig5a & 9 & 2 & 100 & 0.336 & 1.064 & 85.0 \\
\hline Fig5g-i & 60 & 3 & $50 ?$ & 0.343 & 1.088 & 88.4 \\
\hline \multicolumn{4}{|c|}{ Theoretical bulk 2H Si, ref. } & 0.335 & 1.061 & 90 \\
\hline
\end{tabular}

grown on the $\mathrm{Cu}$ grid, and even on the carbon membrane in between the grid bars. We therefore have no real information on the presence of $\mathrm{Cu}$ in the catalysts or in the NWs. The presence of Sn (and also O) was confirmed by EDX in the catalyst particles (see Supplementary information).

\section{Discussion}

The presence of this hexagonal metastable structure obliges us to confront some critical questions: what is creating it and/or what is stabilizing it? Three candidates could be invoked as an answer: (i) the influence of foreign atoms, (ii) kinetic effects during growth and (iii) size effects. We address each of these possible answers in the following.

(i) As we cannot exclude the presence of $\mathrm{Cu}$ in the catalyst, we grew (larger) NWs with $\mathrm{Cu}$ as sole catalyst, to check whether or not this element could promote the $2 \mathrm{H}$ structure in the same growth conditions. However, we found no $2 \mathrm{H}$ regions whatsoever in the NWs grown under such conditions. We therefore have no solid proof that $\mathrm{Cu}$ is a key factor in producing the $2 \mathrm{H}$ phase. On the other hand, $\mathrm{Sn}$ from the catalyst droplets may be present in significant concentrations in the nanowires, as was positively confirmed by atom probe tomography ${ }^{26}$. As a matter of fact, we have shown in previous work that during the plasma-assisted VLS growth of silicon nanowires using Sn as catalyst, a wetting layer should form around the wires in order to stabilize the drop and allow NW growth $^{24}$. The (larger) NWs in that work, although cubic, included a very high density of twins. The presence of such wetting layer around the growing nanowire could play a crucial role in stabilizing the hexagonal structure.

(ii) As the $2 \mathrm{H}$ polytype was shown to form kinetically in GaAs nanowires ${ }^{27}$, it could form in the same way in SiNWs. However, if efficient, this mechanism would also deliver the $2 \mathrm{H}$ structure in larger nanowires, a phenomenon which we have never observed.

(iii) Finally, at these scales, size effects could play a predominant role. The surface-to-volume ratio is increased in small objects, which has consequences that could present themselves in two ways: (a) the surface tension from the sidewalls becomes significant and will exert a strongly anisotropic stress on the nanowire structure that could somehow mimic the cases where anisotropic stress is at the origin of the formation of this phase, and (b) the hexagonal phase would have atomic surface structures with lower energy, so that the global (surface + volume) free energy would be lower ${ }^{28}$. Indeed, two teams have separately calculated that the $2 \mathrm{H}$ phase would be favoured in small nanowires oriented in the [0002] direction ${ }^{28,29}$. However, our own data show no structural difference between the two orientations of the NW presented in fig. 3. Moreover, the fact that we observe the $2 \mathrm{H}$ phase in NWs covered by an amorphous layer (be it thin or thick) tends to indicate that any pristine surface structure plays a minor role. The exact surface structure that develops during growth remains to be determined; in particular, the wetting by the catalyst postulated earlier ${ }^{24}$ could have a significant contribution. 
In previous experimental works, other authors prepared SiNWs of similar sizes with CVD and using $\mathrm{Au}^{30}$ or $\mathrm{Ag}^{31}$ as catalysts, and found them to possess only the cubic structure. The fact that these NWs were not oriented in the [111]/[0002] direction for which calculations have predicted the $2 \mathrm{H}$ phase $^{1,28,29}$ makes them compatible with those calculations. However here, the NW of fig. 3 has a length of more than 60 $\mathrm{nm}$ with [10-10] orientation, which must be stabilised by some other means than those proposed by Akiyama et $a .^{28}$.

Finally, among the parameters that differentiate the present work from previous experiments, we would like to highlight the use of $\mathrm{Sn}$ as catalyst in a plasma environment. Calculations by Akiyama et al. underlined the effect of the surface atomic configuration (associated in their paper with a varying coverage by hydrogen ${ }^{28}$ ), which could switch the phase from cubic to hexagonal and vice versa. Tin, by wetting the NW surfaces $^{24}$, would change their contribution to the global free energy balance. In small NWs with high surface-to-volume ratios, this could switch the equilibrium towards the $2 \mathrm{H}$ hexagonal metastable phase.

\section{Conclusions}

In this paper, we have presented TEM images and diffraction patterns acquired in the $[110]_{3 \mathrm{C}} /[1-210]_{2 \mathrm{H}}$ zone axis of VLS grown silicon nanowires, which cannot be interpreted as the result of a cubic stacking configuration. These are the first unambiguous TEM observations of the $2 \mathrm{H}$ hexagonal $\mathrm{Si}$ phase in as-grown SiNWs. These nanowires differ from those usually studied essentially by their small diameter $(<5 \mathrm{~nm})$ and the fact that we use plasma-assisted CVD and $S n$ as catalyst for their growth. The hexagonal structure appears to be stable, over time in standard ambient temperature and pressure, and upon annealing at $700{ }^{\circ} \mathrm{C}$ in vacuum.

\section{Acknowledgements}

This work was partly supported by the French National Research Agency (ANR) through the projects Solarium (ANR14-CE05-0025), Platofil (ANR-14-CE26-0020) and the TEMPOS Equipex (ANR-10-EQPX-50), pole NanoMAX. We acknowledge the Centre interdiscipinaire de microscopie électronique de I'X (CIMEX) and Pierre-Eugène Coulon for providing TEM equipment in perfect working order at Ecole polytechnique. Jian Tang thanks the "French Ministry for Higher Education and Research" for his PhD funding.

\section{Notes and references}

¥ i.e. with no stress applied other than that of the SiNWs' own surfaces.

1. M. Amato, T. Kaewmaraya, A. Zobelli, M. Palummo and R. Rurali, Nano Lett., 2016, 16, 5694-5700.

2. C. Rödl, T. Sander, F. Bechstedt, J. Vidal, P. Olsson, S. Laribi and J. F. Guillemoles, Phys. Rev. B, 2015, 92, 045207.
3. G. Weill, J. L. Mansot, G. Sagon, C. Carlone and J. M. Besson, Semicond. Sci. Technol., 1989, 4, 280-282.

4. H. Olijnyk, S. K. Sikka and W. B. Holzapfel, Phys. Lett. A, 1984, 103, 137-140.

5. J. Z. Hu, L. D. Merkle, C. S. Menoni and I. L. Spain, Phys. Rev. $B, 1986,34,4679-4684$.

6. R. H. Wentorf and J. S. Kasper, Science, 1963, 139, 338-339.

7. P. Mullner and P. Pirouz, Mater. Sci. Eng., A, 1997, 233, 139144.

8. N. Garcia-Castello, J. D. Prades, R. Orlando and A. Cirera, J. Phys. Chem. C, 2012, 116, 22078-22085.

9. S. Q. Wang and H. Q. Ye, J. Phys.: Condens. Matter, 2003, 15, 5307-5314.

10. D. B. Zhang, M. Hua and T. Dumitrica, J. Chem. Phys., 2008, 128.

11. S. L. Qiu and P. M. Marcus, J. Phys.: Condens. Matter, 2011, 23, 215501.

12. P. Pirouz, R. Chaim, U. Dahmen and K. H. Westmacott, Acta Metall. Mater., 1990, 38, 313-322.

13. Y. Qiu, H. Bender, O. Richard, M.-S. Kim, E. Van Besien, I. Vos, M. de Potter de ten Broeck, D. Mocuta and W. Vandervorst, Sci. Rep., 2015, 5, 12692.

14. H. I. T. Hauge, M. A. Verheijen, S. Conesa-Boj, T. Etzelstorfer, M. Watzinger, D. Kriegner, I. Zardo, C. Fasolato, F. Capitani, P. Postorino, S. Kölling, A. Li, S. Assali, J. Stangl and E. P. A. M. Bakkers, Nano Lett., 2015, 15, 5855-5860.

15. L. Vincent, G. Patriarche, G. Hallais, C. Renard, C. Gardès, D. Troadec and D. Bouchier, Nano Lett., 2014, 14, 4828-4836.

16. H. I. T. Hauge, S. Conesa-Boj, M. A. Verheijen, S. Koelling and E. P. A. M. Bakkers, Nano Lett., 2016.

17. Y. Miyamoto and M. Hirata, J. Phys. Soc. Jpn., 1978, 44, 181190.

18. A. Fontcuberta i Morral, J. Arbiol, J. D. Prades, A. Cirera and J. R. Morante, Adv. Mater., 2007, 19, 1347-1351.

19. J. Arbiol, B. Kalache, P. Roca i Cabarrocas, J. R. Morante and A. Fontcuberta i Morral, Nanotechnology, 2007, 18, 305606.

20. F. Fabbri, E. Rotunno, L. Lazzarini, N. Fukata and G. Salviati, Sci. Rep., 2014, 4, 3603.

21. D. W. Pashley, M. J. Stowell and T. J. Law, Phys. Stat. Sol. b, 1965, 10, 153-163.

22. C. Cayron, M. Den Hertog, L. Latu-Romain, C. Mouchet, C. Secouard, J.-L. Rouviere, E. Rouviere and J.-P. Simonato, J. Appl. Crystallogr., 2009, 42, 242-252.

23. J. Tang, J.-L. Maurice, W. H. Chen, S. Misra, M. Foldyna, E. V. Johnson and P. Roca i Cabarrocas, Nanoscale Res. Lett., 2016, 11, 455.

24. S. Misra, L. Yu, W. Chen and P. Roca i Cabarrocas, J. Phys. Chem. C, 2013, 117, 17786-17790.

25. P. Stadelmann, JEMS-Saas, http://www.jems-saas.ch/).

26. W. Chen, L. Yu, S. Misra, Z. Fan, P. Pareige, G. Patriarche, S. Bouchoule and P. Roca i Cabarrocas, Nat Commun, 2014, 5, 4134.

27. F. Glas, J.-C. Harmand and G. Patriarche, Phys. Rev. Lett., 2007, 99, 146101.

28. T. Akiyama, K. Nakamura and T. Ito, Phys. Rev. B, 2006, 74, 033307.

29. R. Kagimura, R. W. Nunes and H. Chacham, Phys. Rev. Lett., 2005, 95, 115502.

30. Y. Cui, L. J. Lauhon, M. S. Gudiksen, J. Wang and C. M. Lieber, Appl. Phys. Lett., 2001, 78, 2214-2216.

31. J. V. Wittemann, W. Münchgesang, S. Senz and V. Schmidt, J. Appl. Phys., 2010, 107, 096105. 